\title{
Proteolytic queues at ClpXP increase antibiotic tolerance
}

Heather S. Deter ${ }^{+1}$, Alawiah H. Abualrahi ${ }^{\ddagger 1}$, Prajakta Jadhav ${ }^{1}$, Elise K. Schweer ${ }^{1}$, Curtis T. Ogle ${ }^{2}$, and Nicholas C. Butzin*1

${ }^{1}$ Department of Biology and Microbiology. South Dakota State University. Brookings, SD. 57006. USA.

${ }^{2}$ Independent researcher. Prosser, WA, 99350. USA.

${ }^{\ddagger}$ Authors contributed equally

${ }^{*}$ Corresponding author

\section{Supporting Information}


Supplementary Figures and Data

a. Induction in exponential phase

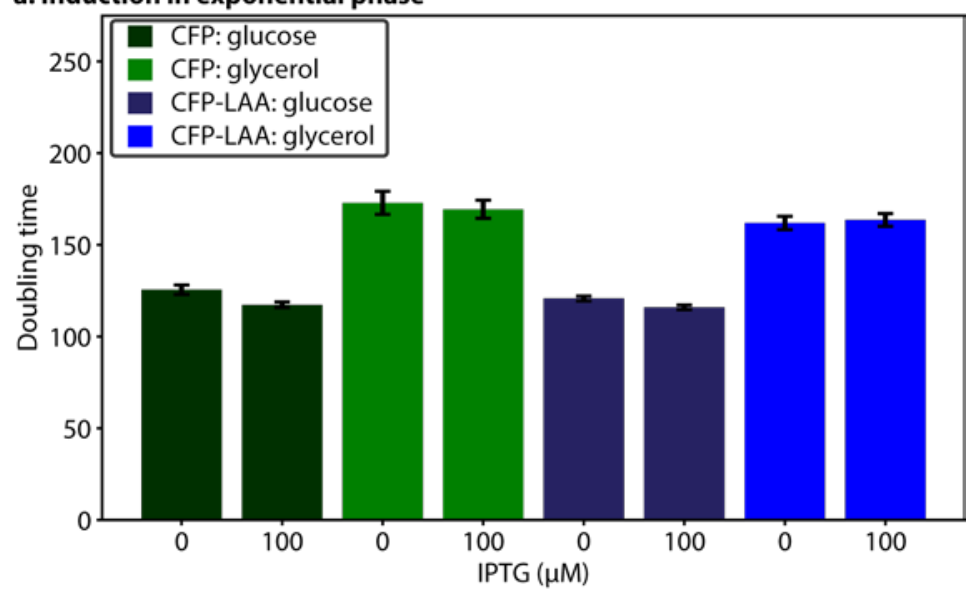

b. Induction upon transition out of stationary phase

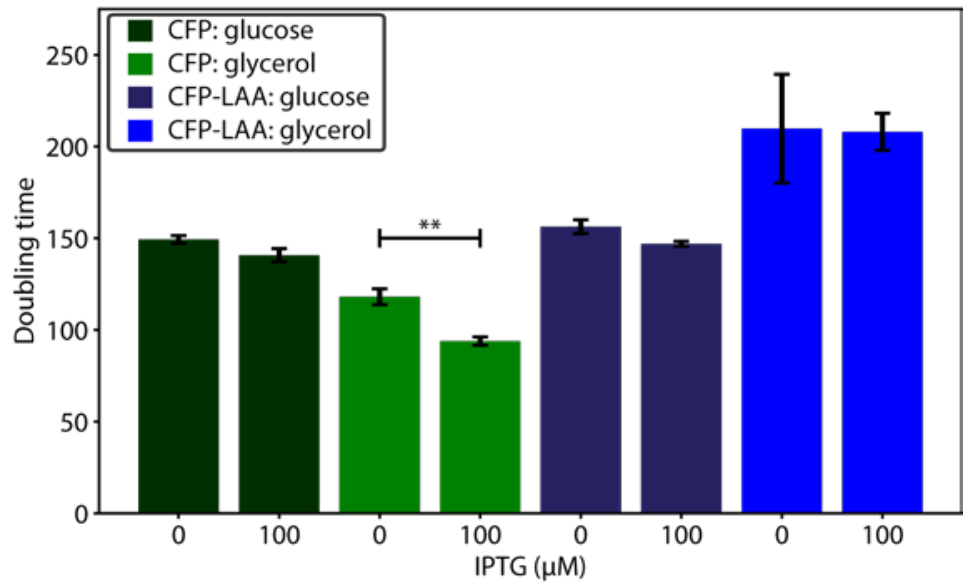

Fig. S1. Induction of untagged CFP and CFP-LAA tag has no apparent effect on growth in MMB+ media. a. Cultures were induced during exponential phase. Induction of CFP-untagged or CFP-LAA had no apparent effect on growth when glucose or glycerol were the sole carbon source. $\mathbf{b}$. Cultures were induced after 1/100 dilution into fresh media (the same dilution used during persister quantification, see Methods). Induction of CFP-untagged or CFP-LAA had no apparent effect on growth when glucose was the sole carbon source.

Induction of CFP-untagged or CFP-LAA had no apparent effect on growth when glycerol was the sole carbon source. CFP-untagged grew slightly faster when induced by IPTG in glycerol media. However, if this difference in growth were to affect antibiotic survival, we would expect a decrease rather than no significant change in survival (see Results). Doubling time was calculated based on $\mathrm{OD}_{600}$ readings over time in a microplate reader (see Methods). $n=4$. Error bars represent SEM. 


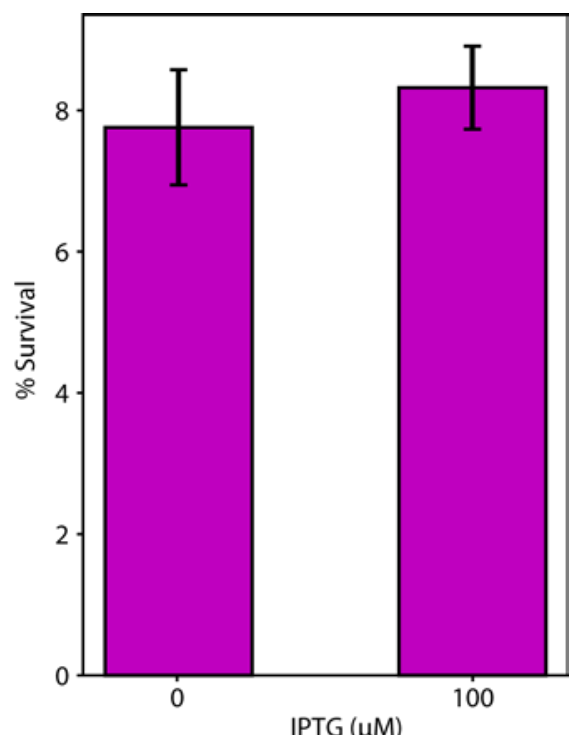

Fig. S2. Induction of CFP-LAA does not increase survival of cells treated with chloramphenicol. Cultures were treated with chloramphenicol, an antibiotic that inhibits translation, after a $1 / 10$ dilution into fresh media from stationary phase. Induction of CFP-LAA via IPTG had no significant change in persistence compared to the uninduced cultures $(\mathrm{p}>0.7 ; \mathrm{n} \geq 3)$. Error bars represent SEM. 
A Typical Persister Assay
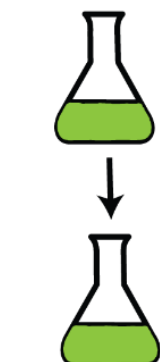

One biological replicate

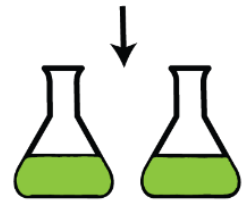

After $24 \mathrm{~h}$, samples were taken

Samples were diluted and plated for quantification.

From this plating, the $\mathrm{CFU} / \mathrm{ml}$ is calculated.
Cultures were incubated in $125 \mathrm{ml}$ flasks at $37^{\circ} \mathrm{C}$, shaking at $250 \mathrm{rpm}$.

At about $\mathrm{OD}_{600 \mathrm{~mm}} 0.3,20 \mathrm{ml}$ of culture was aliquoted into a $125 \mathrm{ml}$ flask, and then incubated for $16 \mathrm{~h}$ to reach stationary phase.

After $16 \mathrm{~h}$, each biological replicate was divided into two $125 \mathrm{ml}$ flasks.

Both flasks contained $0.2 \%$ arabinose and one flask also contained $100 \mathrm{nM}$ IPTG to induce cfp expression.

All flasks were incubated at $37^{\circ} \mathrm{C}$, shaking at $250 \mathrm{rpm}$ for $24 \mathrm{~h}$.

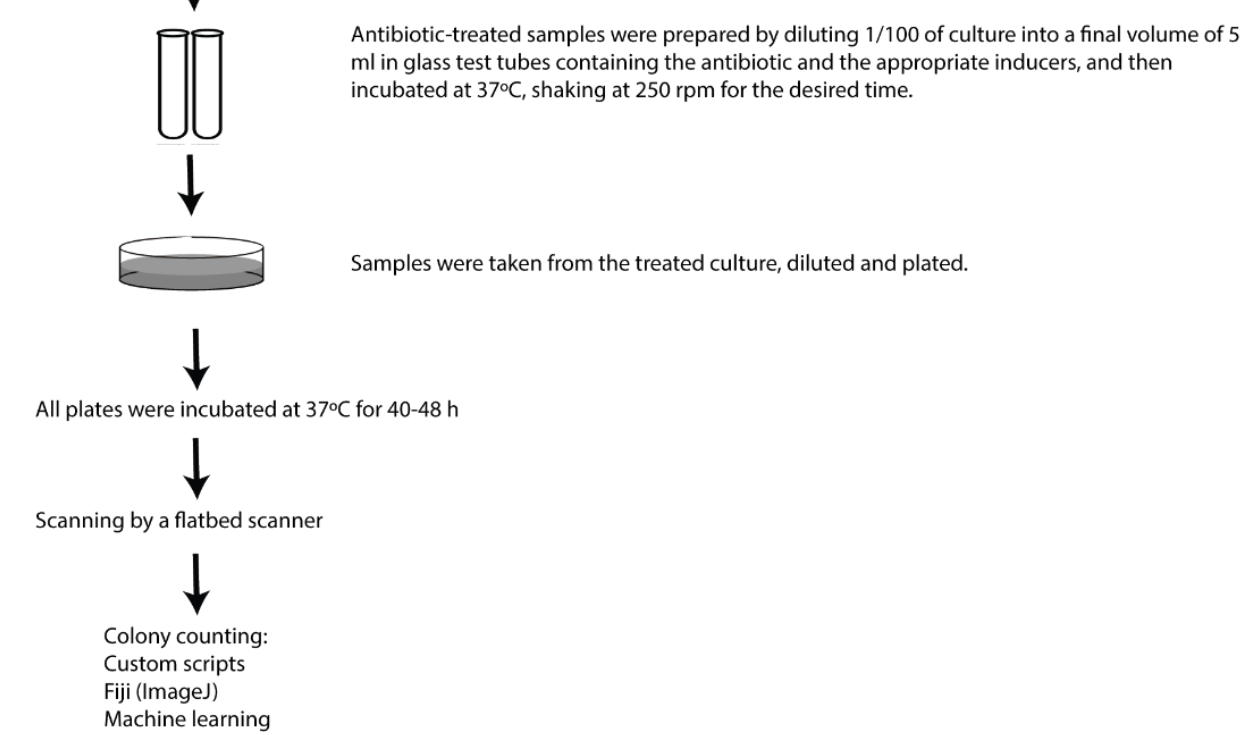

Fig. S3. Persister assay flow chart of a typical assay. See Methods for details. 


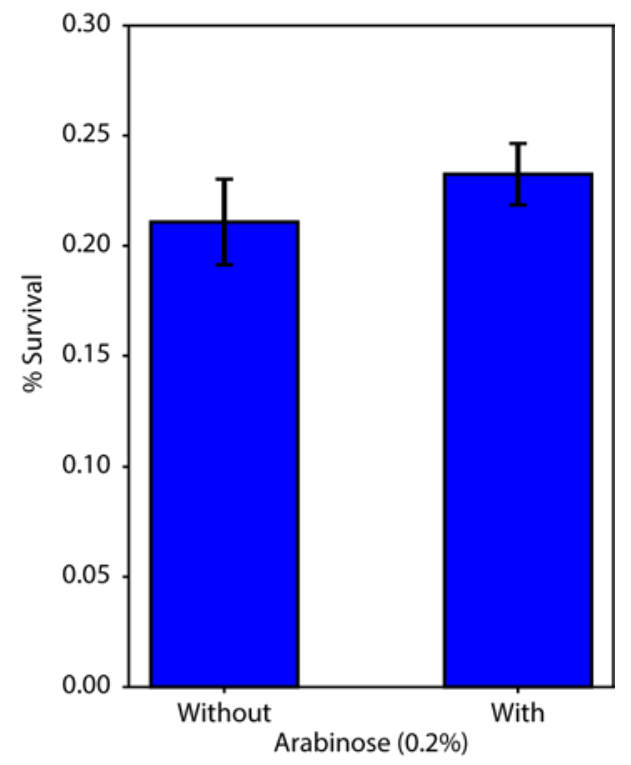

Fig. S4. The addition of arabinose had no apparent effect on the tolerance/persister level during ampicillin treatment. Both IPTG and arabinose are inducers for CFP untagged and CFP-LAA tagged proteins. IPTG induces expression, arabinose alone does not induce expression, but arabinose can enhance expression when used in combination with IPTG. The effect of adding arabinose (0.2\%) on tolerance/persistence to ampicillin was tested with CFP-LAA. Adding arabinose does not have a significant effect on survival of cells after 3 hours of ampicillin treatment ( $\mathrm{p}>0.3$ ). Error bars represent $S E M . \mathrm{N} \geq 3$.

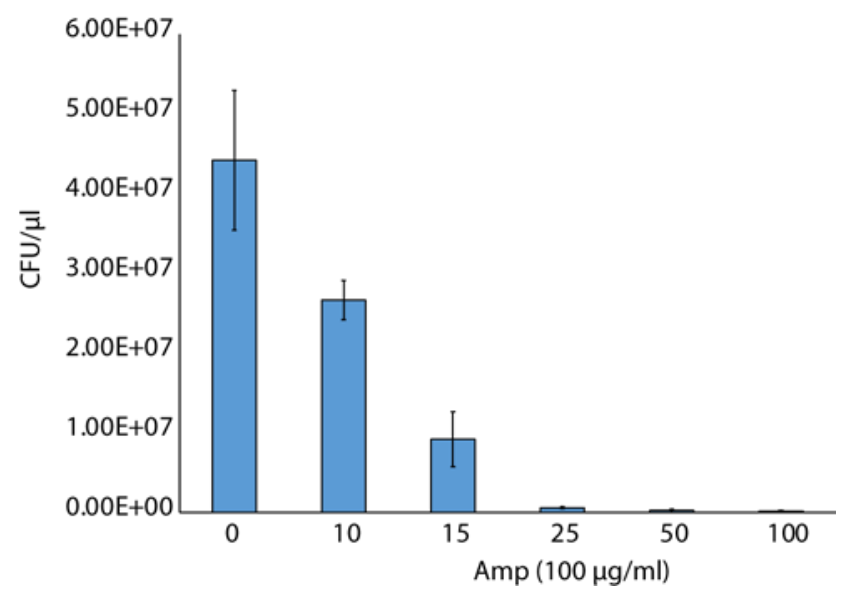

Fig. S5. Determination of Minimal Inhibitory Concentration (MIC) for ampicillin. Exponential phase cultures were treated with different concentrations of ampicillin. The MIC was determined to be $10 \mu \mathrm{g} / \mathrm{ml}$ ( $\mathrm{p}<0.03$ compared to zero). Error bars represent the standard deviation. The ciprofloxacin MIC was determined in a similar manner. 